\title{
Insights into the transcriptome of oenocytes from Aedes aegypti pupae
}

\author{
Gustavo Ferreira Martins ${ }^{1 /+}$, José Marcelo Ramalho-Ortigão², Neil Francis Lobo ${ }^{3}$, \\ David William Severson ${ }^{3}$, Mary Ann McDowell ${ }^{3}$, Paulo Filemon Paolucci Pimenta ${ }^{4}$
}

\author{
'Departamento de Biologia Geral, Universidade Federal de Viçosa, Viçosa, MG, Brasil \\ ${ }^{2}$ Department of Entomology, Kansas State University, Manhattan, Kansas, USA \\ ${ }^{3}$ Department of Biological Sciences, Center for Global Health and Infectious Diseases, University of Notre Dame, Notre Dame, IN, USA \\ ${ }^{4}$ Laboratório de Entomologia Médica, Instituto René Rachou-Fiocruz, Belo Horizonte, MG, Brasil
}

\begin{abstract}
Oenocytes are ectodermic cells present in the fat body of several insect species and these cells are considered to be analogous to the mammalian liver, based on their role in lipid storage, metabolism and secretion. Although oenocytes were identified over a century ago, little is known about their messenger RNA expression profiles. In this study, we investigated the transcriptome of Aedes aegypti oenocytes. We constructed a cDNA library from Ae. aegypti MOYO-R strain oenocytes collected from pupae and randomly sequenced 687 clones. After sequences editing and assembly, 326 high-quality contigs were generated. The most abundant transcripts identified corresponded to the cytochrome P450 superfamily, whose members have roles primarily related to detoxification and lipid metabolism. In addition, we identified 18 other transcripts with putative functions associated with lipid metabolism. One such transcript, a fatty acid synthase, is highly represented in the cDNA library of oenocytes. Moreover, oenocytes expressed several immunity-related genes and the majority of these genes were lysozymes. The transcriptional profile suggests that oenocytes play diverse roles, such as detoxification and lipid metabolism, and increase our understanding of the importance of oenocytes in Ae. aegypti homeostasis and immune competence.
\end{abstract}

Key words: Aedes aegypti - oenocytes - transcriptome - cytochrome P450 - lipid metabolism - detoxification

Aedes aegypti is the major vector of yellow fever and the principal global vector of dengue virus. Different populations of Ae. aegypti vary in their ability, or competence, to transmit a particular pathogen. Vector competence refers to the intrinsic permissiveness of an arthropod vector for infection, replication and transmission of a pathogen to a vertebrate. Vector competence is consistent with a general model in which multiple structural or biochemical factors must be present for the successful completion of a pathogen's life cycle. In some instances, differences in vector competence can be traced to antimicrobial responses to pathogens through the activation of the insect's innate immune system. In disease vectors such as mosquitoes, understanding the regulation of the innate immune response can provide novel insights into vector competence and disease transmission. Innate responses in insects are rapid and transient and the hemolymph, the midgut and the fat body are involved in determining innate immunity in insects (Christophides et al. 2004, Barillas-Mury et al. 2005).

The fat body is the major metabolic organ of insects and the principal site of transcriptional activation and the synthesis of effector molecules such as antimicro-

Financial support: University of Notre Dame (FRP2008 to JMRO), NIAID, NIH (AI059342 to DWS), CNPq, CAPES, PRONEX, FAPEMIG, FIOCRUZ

NFL was supported by NIH/NIAID (HHSN266200400039C).

+Corresponding author: gmartins@ufv.br

Received 1 December 2010

Accepted 24 March 2011 bial peptides (AMPs) that are key players in the innate response against several bacteria and fungi (Tzou et al. 2002, Barillas-Mury et al. 2005, Feitosa et al. 2006). The fat body is a diffuse organ lining the body cavity of insects and consists of two types of cells: trophocytes of mesodermic origin and oenocytes of ectodermic origin. Trophocytes are thought to play a local role in intermediary metabolism by storing lipids, carbohydrates and proteins (Dean et al. 1985, Martins \& Pimenta 2008).

Larval oenocytes were first described in insects over 140 years ago. Since then, their function has remained unclear and they have been investigated in largely descriptive studies implicating larval oenocytes in processes such as cuticle synthesis and the regulation of hemolymph composition (reviewed by Dean et al. 1985). Gutierrez et al. (2007) demonstrated that oenocytes from Drosophila melanogaster larva store lipid as cytoplasm droplets and appear to play a crucial role in insect development. Additionally, D. melanogaster oenocytes and the mammalian liver share several homologous genes coding for lipid metabolism/processing. Of the 51 transcripts that were identified to be exclusive to $D$. melanogaster larvae oenocytes, $40 \%$ of the transcripts have human homologues that are associated with lipid metabolism and protein processing. Thus, in Drosophila, oenocytes can be considered analogous to mammalian hepatocytes (Gutierrez et al. 2007). Recently, WickerThomas et al. (2009) demonstrated that the knock-down of desaturase genes (desatI and desatF) in oenocytes suppresses cuticular sex pheromones biosynthesis, affecting the courtship behaviour in D. melanogaster.

Despite the completion of whole genome sequencing for three mosquito species (Anopheles gambiae, Ae. aegypti, and Culex quinquefasciatus) the global gene ex- 
pression profile (transcriptome) in mosquito oenocytes is currently unknown. One role assigned to An. gambiae oenocytes is the production of nicotinamide adenine dinucleotide phosphate-oxidase (NADPH) cytochrome P450 reductase (CPR), an enzyme required for the activation of cytochrome P450 that is involved in insecticide resistance in mosquitoes (Lycett et al. 2006).

In this study, we have constructed a cDNA library of oenocytes that were dissected from an Ae. aegypti substrain, MOYO-R, and selected for refractoriness to infection by the avian malarial parasite, Plasmodium gallinaceum (Thathy et al. 1994). The substrain is also resistant to infection with dengue 2 virus (DENV2) (Schneider et al. 2007). Random sequencing of 687 clones revealed an expression profile that is compatible with the important physiological roles believed to be played by oenocytes in Ae. aegypti. Our study is the first analysis of the transcriptome of these cells in mosquitoes and provides significant insights into the potential role of oenocytes in lipid metabolism, detoxification and innate immunity responses.

\section{MATERIALS AND METHODS}

Mosquitoes - Origin and selection criteria for the Ae. aegypti MOYO-R substrain are described elsewhere (Thathy et al. 1994). The original substrain was lost and a similar substrain was reselected prior to the present study. Mosquitoes were reared in an environmental chamber held at $26^{\circ} \mathrm{C}$ and $85 \%$ relative humidity with 16-h light/8-h dark cycles that included a $1 \mathrm{~h}$ crepuscular period at the beginning and end of each cycle. Larvae were reared on a dried bovine liver powder suspension. Adults were provided with a $2 \%$ sugar solution ad libitum. The colony was maintained by allowing adult females to blood feed on anaesthetised rats. Our protocol for maintenance and care of experimental animals was reviewed and approved by the Institutional Animal Care and Use Committee at the University of Notre Dame.

Dissection and oenocyte collection - Pupae were transferred to Petri dishes containing $1 \mathrm{X}$ phosphate buffered saline and dissected under a Zeiss stereoscope microscope. Pupae were chosen for oenocytes collection because in this developmental stage the fat body is rebuilt and oenocytes are organised in loose cells in the organ. In adults and larvae, oenocytes are individualised cells that are spread between trophocytes, which makes it difficult to separate the oenocytes without contamination from other cells (Martins et al. 2011a). Fat bodies were excised from the hemocoel and the oenocytes were mechanically separated by gentle agitation. Under the stereoscope, oenocytes were easily recognised as isolated large cells or clusters. Unlike trophocytes, the oenocytes are larger and do not have cytoplasm filled with lipid droplets. In addition, lipid droplets in trophocytes appear bright under stereoscope light, which makes it easier to separate trophocytes from oenocytes (Martins et al. 2011a). Oenocytes were collected and transferred to microcentrifuge tubes containing $10 \mu \mathrm{L}$ RNAlater Solution (Ambion, Austin, TX). Up to 100 oenocytes were collected per sample. Following a brief spin, samples were kept at $-70^{\circ} \mathrm{C}$ until messenger RNA (mRNA) extraction. Morphological observations of dissected oenocytes by Giemsa staining or scanning electron microscopy analysis confirmed that all collected cells were oenocytes.

$m R N A$ extraction and cDNA library construction mRNA was purified with the Micro-FastTrack mRNA 2.0 isolation kit (Invitrogen, Carlsbad, CA) and used to synthesise the first strand of cDNA. A cDNA library, enriched for full-length cDNA, was synthesised using the SMART cDNA library construction kit (Clontech, Mountain View, CA). One microgram of double-stranded DNA from the original library was fractionated using a Chromaspin 1000 column (Clontech, Mountain View) into small (S), medium (M) and large (L) transcripts based on their electrophoresis profile on a $1.1 \%$ agarose gel. Pooled fractions from each of the fractionated samples ( $\mathrm{S}, \mathrm{M}$ and $\mathrm{L}$ ) were individually ligated into a Lambda TriplEx2 vector (Clontech, Mountain View) and packaged into a lambda phage using Gigapack III, Gold package extract (Stratagene, La Jolla, CA). Individual libraries were plated on lysogeny broth agar plates to achieve roughly $30-60$ plaques per $70-\mathrm{mm}$ plates.

Sequencing - Sequencing of randomly selected clones was performed as previously described (Ramalho-Ortigão et al. 2007). Briefly, single, isolated plaques were picked from the plates using sterile wooden sticks and placed into $100 \mu \mathrm{L}$ of water. Each cDNA was amplified by polymerase chain reaction (PCR) using Platinum PCR SuperMix (Invitrogen, Carlsbad), $4 \mu \mathrm{L}$ of the template (from the $100 \mu \mathrm{L}$ ) and vector-specific primers PT2F1 (5'-AAGTACTCTAGCAATTGTGAGC-3') and PT2R1 (5'-CTCTTCGCTATTACGCCAGCTG-3'). The PCR amplification products were washed in $150 \mu \mathrm{L}(2 \mathrm{X}) \mathrm{DNAse} / \mathrm{RNAse}$ free water in Multiscreen PCR cleaning plates (Millipore, Billerica, MA) by applying vacuum $(10 \mathrm{psi})$ and suspended in $50 \mu \mathrm{L}$ of DNAse/RNAse free water. After cleaning, PCR products were cycle sequenced with the PT2F3 (5'-TCTCGGGAAGCGCGCCATTGT-3') primer using BigDye 3.1 (Applied Biosystems, Foster City, CA). Sequencing reaction products were cleaned using a Multiscreen PCR cleaning plate (Millipore, Billerica, MA) and analysed using the ABI3730XL automated sequencer (Applied Biosystems, Foster City).

Sequence analyses - Seqman (DNAstar) software was used to trim the vector sequences and low-quality sequences and assemble the resulting high-quality sequences into contigs using $96 \%$ identity. Each contig was manually examined and those sequences with less than $80 \mathrm{bp}$ were removed from the analysis. Contigs and singletons (contigs containing only 1 sequence) were compared to Ae. aegypti Genebuild V1.1 (AaegL1.1) using VectorBase (www.vectorbase.org). InterPro (www.ebi.ac.uk/interpro/) and Gene Ontology (www.geneontology.org/) were used to assign function to Ae. aegypti transcripts. Various types of meta-data, including gene transcript information, predicted gene products, protein domains and indication of biological function were also manually added. 


\section{RESULTS AND DISCUSSION}

As shown in Fig. 1, several clusters of dissected oenocytes were obtained from the fat body of Ae. aegypti MOYO-R pupae. Giemsa staining revealed a single cell type that was primarily organised in clusters. Clustering of dissected oenocytes may be associated with cell behaviour, as these cells are known to organise in such a manner on the fat bodies of Ae. aegypti pupae (Martins et al. 2011a).

Approximately 2,500 oenocytes obtained from clusters or as individual cells were used to construct a fractionated cDNA library. Following the random sequencing of 687 clones, 503 transcripts [expressed sequence tags (ESTs)] were edited and assembled into 326 contigs. Only those transcripts displaying similarity to sequences previously identified in the Ae. aegypti genome (cut-off of E-05) were included in the analysis. The ESTs identified here were deposited in dbEST under accessions FE425950-FE426452. For a selected number of transcripts, we also provided the corresponding accession numbers assigned by the Ae. aegypti genome project (given by the code AAEL00000) to facilitate matching transcripts identified in this study with gene sequences previously identified by the Ae. aegypti whole genome sequence. This work constitutes the first transcriptome analysis of mosquito oenocytes and, in the case of $A e$. aegypti, provides experimental evidence for various hypothetically transcribed genes.

The relative transcript abundance of Ae. aegypti oenocytes was assumed from transcript density (the library was not normalised) and the abundance is summarised in Fig. 2. About $22.1 \%$ of transcripts identified were associated with known functions and/or physiological roles believed to be performed by oenocytes. Transcripts identified in our analyses were distributed as follows: $8 \%$ of the transcripts coded for five distinct cytochrome P450

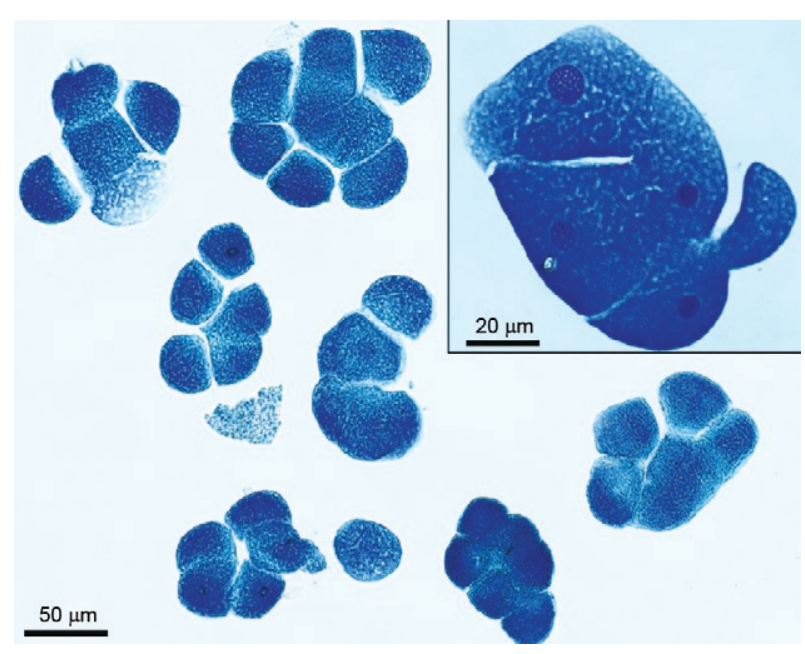

Fig. 1: Giemsa stain of oenocytes dissected from Aedes aegypti pupae. Oenocytes are shown as clusters of 3-8 cells with outline of each cell easily detectable. Inset, cluster of four oenocytes displaying individual nuclei and abundant canaliculi. superfamily protein members, $4.2 \%$ encoded other types of detoxification proteins, such as alcohol dehydrogenase and catalase, $8 \%$ encoded lipid-metabolising proteins, such as fatty acid synthase, elongase and estradiol 17 beta-dehydrogenase, and $1.9 \%$ encoded proteins that likely participate in mechanisms of innate immune response, such as lysozymes and serine protease (Table).

Of the total transcripts, $18 \%$ of the transcripts were predicted to encode ribosomal genes, $8 \%$ to encode nuclear metabolism proteins (i.e., topoisomerases, transcription factors and nucleases) and $9 \%$ to encode proteins involved in processing and degradation, including eight transcripts coding for protein associated with the proteosome, in addition to lysosomes, cathepsins and ubiquitins (Fig. 2). Although pupae are considered to be a transitional stage with significant tissue reorganisation, few transcripts related to protein synthesis and metabolism were identified. However, this was likely due to the random nature of the analysis.

Four percent of the transcripts encoded proteins with roles in energy metabolism (i.e., NADH dehydrogenase and cytochrome oxidase $\mathrm{C}$ that were both involved in the respiratory chain and ATPase), 2.1\% encoded proteins that participate in cell transport systems, $4.7 \%$ encoded cell signalling proteins and $1.9 \%$ encoded vesicular trafficking proteins (Fig. 2). Approximately 19\% of the sequences corresponded to genes without known function.

Proteins that had putative functions differing from the categories listed above were grouped as proteins with miscellaneous function and accounted for $7.5 \%$ of all sequences. Transcripts without similarity to known genes were grouped under "no hits found" and represented $1.9 \%$ of all sequences (Fig. 2). Below is a more detailed description of some of the transcripts identified in this study and their putative roles in Ae. aegypti oenocytes.

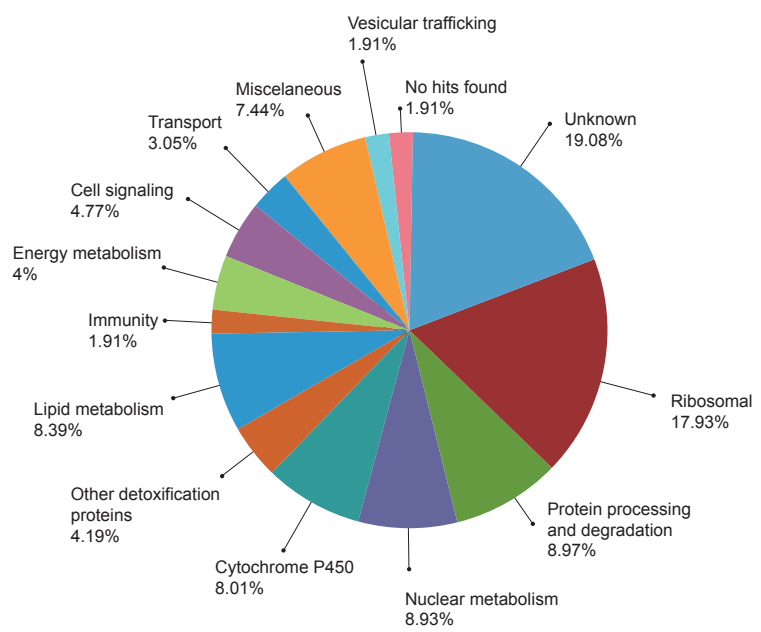

Fig. 2: relative transcript distribution of an Aedes aegypti oenocyte cDNA library. Putative protein functions were assigned according to homology to known genes. No hits transcripts were matched to sequences included in Ae. aegypti supercontigs deposited with VectorBase. No hits found represent those transcripts from our analyses without a match to known genes. 
Cytochrome P450 - Transcripts coding for cytochrome P450s (P450) were highly represented in the Ae. aegypti oenocyte transcriptome. We identified 42 transcripts ( $8 \%$ of the total sequences) corresponding to at least three putative proteins representing different $\mathrm{P} 450$ genes and one transcript predicting a protein with a $\mathrm{P} 450$ domain (Table). The transcripts that were identified as P450 correspond to genes identified by the Ae. aegypti genome project and were deposited in GenBank under the accessions AAEL006824, AAEL004054, AAEL009124 and AAEL009699. Interestingly, the latter sequence was identified only as a hypothetical protein by the Ae. aegypti whole genome sequence. Thus, our study provides experimental evidence for the expression of predicted and/or hypothetical genes.

Cytochrome P450s are a large superfamily of hemoproteins found in bacteria, archaea and eukaryotes with as many as 450 genes per single genome. In insects, more than $660 \mathrm{P} 450$ genes distributed between a wide number of families and subfamilies have been characterised in genome sequencing projects and other projects associated with mechanisms of insecticide resistance (Li et al. 2007, Komagata et al. 2010). P450 genes are expressed in a tissue-specific manner, indicating their diverse physiological functions (Simpson 1997). In mammals, P450s are commonly found in hepatocyte microsomes, smooth endoplasmic reticulum and the mitochondria of steroidogenic cells (Simpson 1997). Additionally, different forms of P450s participate in the metabolism of different molecules, such as sterols, steroid hormones and several lipids that include eicosanoids, vitamin D3 and retinoid (Simpson 1997). In insects, P450s also participate in a variety of physiological roles ranging from development, feeding and growth to resistance to pesticides and tolerance to plant toxins (Scott \& Wen 2001, Gilbert 2004). P450s are intimately involved in the synthesis and degradation of hormones and pheromones, such as 20-hydroxyecdysone and juvenile hormone (Scott \& Wen 2001, Gilbert 2004). P450s expressed in oenocytes might participate in steroid metabolism, as previous data has demonstrated that oenocytes isolated from Tenebrio molitor larvae can synthesise both $\alpha$ and $\beta$ ecdysone from $4-{ }^{14} \mathrm{C}$-cholesterol precursors in vitro (Romer et al. 1974). However, the association between P450 and ecdysone synthesis in oenocytes remains to be elucidated.

In addition to their role in steroid metabolism and in analogy to mammalian hepatocytes, insect oenocytes participate in detoxification processes specifically related to xenobiotics (Sundseth et al. 1990, Gutierrez et al. 2007). In insects, increased transcription of P450s is frequently associated with resistance to insecticides (Scott \& Wen 2001). High expression of P450s has been linked to insecticide resistance in Drosophila (Sundseth et al. 1990) and An. gambiae (Müller et al. 2007, Djouaka et al. 2008).

There is a high degree of similarity between the $A e$. aegypti and An. gambiae proteomes. Approximately 2,000 orthologous proteins are shared between An. gambiae and Ae. aegypti and may represent functions central to mosquito biology. In Ae. aegypti, approximately 8\% of these proteins are members of the P450 family. These data represented an expansion of $\mathrm{P} 450$ gene expression in comparison to Drosophila and Anopheles that could potentially enable a more elaborate detoxifying system and contribute to the overall robustness associated with Aedes in comparison to other species (Nene et al. 2007). An expansion of P450s gene families in the recent Cux. quinquefasciatus genome project was suggested to be reflective of adaptations of this mosquito to polluted larval habitats and this expansion may also render this species particularly adaptable to evasion of insecticide-based control programs (Arensburger et al. 2010).

Other detoxification genes - In addition to cytochrome P450s, 23 other transcripts $(4.2 \%$ of the total sequences) coding for proteins with putative functions associated with detoxification were identified. These transcripts also corresponded to sequences identified in the Ae. aegypti whole genome sequence. Some of these transcripts coded for proteins displaying alcohol dehydrogenase activity, such as quinone oxydoreductase (corresponding to Ae. aegypti whole genome sequence AAEL010668), with a total of 10 sequences found in the library, glucose dehydrogenase (corresponding to $A e$. aegypti whole genome sequence AAEL004027) and a short-chain dehydrogenase (corresponding to Ae. aegyp$t i$ whole genome sequence AAEL008159) (Table). In addition, some of these transcripts also coded the following proteins: a catalase (corresponding to Ae. aegypti whole genome sequence AAEL013407), whose role is possibly linked to the removal of excessive reactive oxygen intermediates that allows the cells to maintain homeostasis following oxidative bursts (Gutierrez et al. 2007), a NADPH CPR (corresponding to Ae aegypti whole genome sequence AAEL003349), that is known to be involved with insecticide clearance in An. gambiae (Lycett et al. 2006), and cytochrome b5 (corresponding to Ae. aegypti whole genome sequence AAEL010017) (Table), that is an important donor of the regulating substrate that binds to P450 with CPR (Scott \& Wen 2001).

Lipid metabolism - Several morphological studies have suggested that oenocytes play active roles during lipid synthesis due to the presence of a developed smooth endoplasmic reticulum. Previous data have also suggested that oenocytes are able to produce integument hydrocarbons (Fan et al. 2003). This lipid processing role was demonstrated in D. melanogaster by Gutierrez et al. (2007) with the identification of 51 genes related to lipid metabolism that were expressed exclusively in the oenocytes of the fruit fly. Interestingly, $40 \%$ of those genes were homologous to human genes related to lipid metabolism/processing.

We identified 23 transcripts that code for proteins with putative functions related to lipid synthesis in the Ae. aegypti oenocytes (Table and Supplementary data). Fatty acid synthase (FAS) was the most represented transcript of this group (10 sequences), followed by two distinct elongases (9 sequences) and an estradiol $17 \beta$-dehydrogenase ( 3 sequences) (Table). The FAS transcripts identified correspond to the Ae. aegypti whole genome sequence AAEL002204, the two elongases correspond to AAEL013542 and AAEL008219 and the es- 
TABLE

cDNAs identified from the expression library generated from oenocytes of Aedes aegypti pupae. Transcripts putatively involved in detoxification, lipid metabolism and innate immunity

\begin{tabular}{|c|c|c|c|}
\hline Accession & Protein & $\begin{array}{c}\text { ESTs } \\
\text { (n) }\end{array}$ & Domain \\
\hline \multicolumn{4}{|c|}{ Detoxification genes } \\
\hline AAEL006824 & Cytochrome P450 & 28 & - \\
\hline AAEL004054 & Cytochrome P450 & 12 & - \\
\hline AAEL009124 & Cytochrome P450 & 1 & - \\
\hline AAEL009699 & Hypothetical protein & 1 & Cytochrome P450 \\
\hline \multicolumn{4}{|c|}{ Other detoxification genes } \\
\hline AAEL010668 & Quinone oxidoreductase & 10 & Alcohol dehydrogenase \\
\hline AAEL013407 & Catalase & 3 & - \\
\hline AAEL003349 & NADPH cytochrome $\mathrm{P} 450$ reductase & 1 & - \\
\hline AAEL010017 & Cytochrome b5 & 1 & - \\
\hline AAEL004027 & Glucose dehydrogenase & 1 & Alcohol oxidase \\
\hline AAEL011314 & Epoxide hydrolase & 1 & - \\
\hline AAEL008159 & Short-chain dehydrogenase & 1 & Alcohol dehydrogenase \\
\hline AAEL009436 & Conserved hypothetical protein & 1 & Heavy metal transport \\
\hline AAEL001134 & Methylmalonate-semialdehyde dehydrogenase & 1 & Aldehyde dehydrogenase \\
\hline AAEL010777 & Thioredoxin, putative & 1 & - \\
\hline AAEL008136 & Hypothetical protein & 1 & Heme oxygenase \\
\hline \multicolumn{4}{|c|}{ Other lipid-metabolism genes } \\
\hline AAEL002204 & Fatty acid synthase & 10 & - \\
\hline AAEL013542 & Elongase, putative & 5 & - \\
\hline AAEL008219 & Elongase, putative & 4 & - \\
\hline AAEL007023 & Estradiol $17 \beta$-dehydrogenase & 3 & - \\
\hline AAEL008144 & Amp dependent ligase & 2 & - \\
\hline AAEL012698 & ATP-binding cassette sub-family A member 3 , putative & 2 & AAA atpase \\
\hline AAEL009214 & Diazepam binding inhibitor, putative & 2 & Acyl-coa-binding protein \\
\hline AAEL013574 & Apolipoprotein D, putative & 2 & - \\
\hline AAEL003125 & Acyl-coa dehydrogenase & 1 & - \\
\hline AAEL008789 & Apolipophorin-III, putative & 1 & - \\
\hline AAEL010405 & Alkyldihydroxy acetonephosphate synthase & 1 & - \\
\hline AAEL004341 & Alpha-esterase & 1 & - \\
\hline AAEL009634 & Steroid dehydrogenase & 1 & - \\
\hline AAEL007283 & Acetyl-coa synthetase & 1 & - \\
\hline AAEL005997 & Allergen, putative & 1 & Cytosolic fatty-acid binding \\
\hline AAEL011676 & Amp dependent coa ligase & 1 & - \\
\hline AAEL009038 & Prolylcarboxypeptidase, putative & 1 & - \\
\hline AAEL003611 & Stearoyl-coa desaturase & 1 & - \\
\hline AAEL002533 & Hypothetical protein & 1 & Male sterility protein \\
\hline AAEL000615 & Hypothetical protein & 1 & Tubby protein \\
\hline AAEL004278 & Hypothetical protein & 1 & Fatty acid desaturase \\
\hline AAEL004120 & Niemann-pick type C-2, putative & 1 & - \\
\hline \multicolumn{4}{|l|}{ Immunity genes } \\
\hline AAEL015404 & Lysozyme $\mathrm{P}$, putative & 6 & - \\
\hline AAEL005753 & Serine protease & 2 & - \\
\hline AAEL013434 & Spatzle 1A & 1 & - \\
\hline AAEL008473 & Cysteine-rich venom protein, putative & 1 & Pathogenesis-related proteins \\
\hline
\end{tabular}

accessions listed are those assigned to genes previously identified via the whole genome sequence of Ae. aegypti. Expressed sequence tags (ESTs) identified in this study were deposited in dbEST under accessions FE425950-FE426452. Protein indicates putative protein coded by transcript. Number of ESTs corresponds to the number of sequences that comprise each contig (contigs were determined as indicated in Materials and Methods). Domain indicates the presence of at least one domain associated with a predicted function. 
tradiol 17ß-dehydrogenase correspond to AAEL007023. Lipid biosynthesis depends on FAS activity in the fat body, as demonstrated by the cricket Gryllus bimaculatus (Lorenz \& Anand 2004). FAS was also shown to be responsible for synthesising long and very-long-chain integument fatty acids in association with elongase and P450 (reviewed by Juárez \& Fernández 2007). Elongase activity is correlated with very-long-chain fatty acids and pheromone synthesis in D. melanogaster (Chertemps et al. 2007) and cuticular long-chain hydrocarbons synthesis in Blatella germanica (Blattaria) (Juárez 2004) and Triatoma infestans (Heteroptera) (reviewed by Juárez \& Fernández 2007). In addition, the integumental oenocytes were shown to be responsible for hydrocarbon synthesis in B. germanica (Fan et al. 2003). The 17 $\beta$-hydroxysteroid dehydrogenases are a group of enzymes that are involved in the interconversion of active and inactive forms of androgens and estrogens in the human liver (Deyashiki et al. 1995).

The transcript abundance and representation obtained from the Ae. aegypti oenocytes cDNA library support our data on the morphology of these cells. In $A e$. aegypti pupae oenocytes, the cytoplasm is almost completely filled by a well-developed smooth endoplasmic reticulum (Martins et al. 2011a), which was shown to be associated with lipid metabolism in other insects such as Rhodnius prolixus (Heteroptera), Calpodes ethlius (Lepidoptera) and B. germanica (Wigglesworth 1988, Jackson \& Locke 1989, Fan et al. 2003).

Innate immunity - In insects, the fat body is the primary organ involved in the response to invading microorganisms. However, for a defence response to be initiated, the pathogen has to be detected by a recognition system that is based on the synthesis of pattern-recognition proteins in the fat body that are also released into the hemolymph (Barillas-Mury et al. 2005). The relatively large size and position of the fat body inside the insect's body cavity allows the fat body to be a powerful organ that is able to secrete AMPs that can rapidly reach effective concentrations against invasive organisms (Barillas-Mury et al. 2005).

Our data indicate that oenocytes constitutively express a number of immune-related genes. The most abundant transcript identified in this category was lysozyme $\mathrm{P}$, with a total of six sequences. This transcript corresponds to the previously identified Ae. aegypti genome sequence accession AAEL015404. Other transcripts correlated with pathogen recognition and the activation of innate immune response are Spatzle (SPZ) 1A (corresponding to Ae. aegypti whole genome sequence AAEL013434) and a serine protease (corresponding to Ae. aegypti whole genome sequence AAEL005753) (Table).

The Toll immune signalling pathway is initiated upon the proteolytic cleavage of SPZ, which then binds to and activates the Toll-like receptor (TLR). Activation of the TLR leads to the degradation of a repressor protein (cactus/IkB) and the nuclear translocation of the transcriptional activator REL1/NF-kB. The transcriptional activator REL1 induces the expression of several effector antimicrobial molecules such as drosomycin. Cleavage of SPZ occurs in the hemocoel by the action of serine proteases, whereas binding to the TLR and activation of the pathway leading to the expression of the AMPs occurs in the fat body (Barillas-Mury et al. 2005).

In Ae. aegypti, Toll5A and SpzlC (homologues of Drosophila TLR and SPZ) were suggested to have a major role in the fat body specific activation of the Toll/ REL1 immune pathway (Shin et al. 2006). Involvement of the Toll signalling pathway in the early stages of the antidengue defence in Ae. aegypti was recently confirmed by knock-down studies of the key negative regulator of the pathway, cactus (Ramirez \& Dimopoulos 2010). In cactus knock-down mosquitoes, DENV titres were significantly reduced. In contrast, for mosquitoes whose innate responses were silenced by the RNAi-induced knockdown of MyD88, DENV titers were elevated.

Approximately $2 \%$ of the transcripts from the fat body of Ae. aegypti vitellogenic females corresponded to proteins related to the immunity, such as cecropins, defensins and lectins (Feitosa et al. 2006). These transcripts were not detected in our analyses of oenocytes. Of interest, Feitosa et al. (2006) did not separate trophocytes from oenocytes. Thus, some of the discrepancies regarding transcripts identified by Feitosa et al. (2006) and the current study may be associated with the fact that oenocytes represent only $5-8 \%$ of the adult mosquito fat body (Martins et al. 2011b). These data underscore the need for cell-based transcriptome analyses. Nevertheless, as differentially expressed immune response-related genes were identified in both cell types, these cells are likely involved with innate responses.

A previous study demonstrated that oenocytes share the network of smooth endoplasmic reticulum with mammal steroidogenic cells and have cell membrane invaginations designed to increase cell surface (Jackson $\&$ Locke 1989). In addition, oenocytes are located in the fat body periphery in the adult Ae. aegypti (Martins et al. 2011a, b). Collectively, these results point to an evolutionary mechanism to enhance the detoxification as well as uptake and secretion of lipids (Gutierrez et al. 2007). Moreover, the peripheral position of oenocytes may facilitate rapid pathogen recognition with the activation of the innate response leading to expression and secretion of defence molecules.

Oenocytes are some of the least studied cells in insects and were considered enigmatic until recently. The oenocyte transcript constitution opened new avenues for understanding the functions of Ae. aegypti oenocytes. According to our results, the oenocytes functions can be defined as detoxifying and lipid metabolising cells (Fan et al. 2003) and secondarily as immune competent cells.

The data presented here represent the first analysis of an oenocyte transcriptome from an invertebrate. This analysis provides a road map for future investigations that aim to elucidate underlying molecular interactions between Ae. aegypti and pathogens transmitted by this mosquito. Future studies will focus on comparative analyses between Ae. aegypti strains that are resistant or susceptible to DENV-2 with a goal of increasing our understanding of the role oenocytes may play in determining vector competence. 


\section{REFERENCES}

Arensburger P, Megy K, Waterhouse RM, Abrudan J, Amedeo P, Antelo B, Bartholomay L, Bidwell S, Caler E, Camara F, Campbell CL, Campbell KS, Casola C, Castro MT, Chandramouliswaran I, Chapman SB, Christley S, Costas J, Eisenstadt E, Feschotte C, Fraser-Liggett C, Guigo R, Haas B, Hammond M, Hansson BS, Hemingway J, Hill SR, Howarth C, Ignell R, Kennedy RC, Kodira CD, Lobo NF, Mao C, Mayhew G, Michel K, Mori A, Liu N, Naveira H, Nene V, Nguyen N, Pearson MD, Pritham EJ, Puiu D, Qi Y, Ranson H, Ribeiro JM, Roberston HM, Severson DW, Shumway M, Stanke M, Strausberg RL, Sun C, Sutton G, Tu ZJ, Tubio JM, Unger MF, Vanlandingham DL, Vilella AJ, White O, White JR, Wondji CS, Wortman J, Zdobnov EM, Birren B, Christensen BM, Collins FH, Cornel A, Dimopoulos G, Hannick LI, Higgs S, Lanzaro GC, Lawson D, Lee NH, Muskavitch MA, Raikhel AS, Atkinson PW 2010. Sequencing of Culex quinquefasciatus establishes a platform for mosquito comparative genomics. Science 330: 86-88.

Barillas-Mury C, Paskewitz S, Kanost MR 2005. Immune responses of vectors. In WC Marquardt, Biology of disease vectors, Elsevier, Amsterdan, p. 363-376.

Chertemps T, Duportets L, Labeur C, Ueda R, Takahashi K, Saigo K, Wicker-Thomas C 2007. A female-biased expressed elongase involved in long-chain hydrocarbon biosynthesis and courtship behavior in Drosophila melanogaster. Proc Natl Acad Sci USA 104: 4273-4278.

Christophides GK, Vlachou D, Kafatos FC 2004. Comparative and functional genomics of the innate immune system in the malaria vector Anopheles gambiae. Immunol Rev 198: 127-148.

Dean RL, Locke M, Collins JV 1985. Structure of fat body. In GA Kerkut, LI Gilbert, Comprehensive insect physiology biochemistry and pharmacology, Pergamon Press, Oxford, p. 77-113.

Deyashiki Y, Ohshima K, Nakanishi M, Sato K, Matsuura K, Hara A 1995. Molecular cloning and characterization of mouse estradiol 17 beta-dehydrogenase (A-specific), a member of the aldoketoreductase family. J Biol Chem 270: 10461-10467.

Djouaka RF, Bakare AA, Coulibaly ON, Akogbeto MC, Ranson H, Hemingway J, Strode C 2008. Expression of the cytochrome P450s, CYP6P3 and CYP6M2 are significantly elevated in multiple pyrethroid resistant populations of Anopheles gambiae s.s. from southern Benin and Nigeria. BMC Genomics 9: 538.

Fan Y, Zurek L, Dykstra MJ, Schal C 2003. Hydrocarbon synthesis by enzymatically dissociated oenocytes of the abdominal integument of the German cockroach Blatella germanica. Naturwissenschaften 90: 121-126.

Feitosa FM, Calvo E, Merino EF, Durham AM, James AA, de Bianchi AG, Marinotti O, Capurro ML 2006. A transcriptome analysis of the Aedes aegypti vitellogenic fat body. J Insect Sci 6: 1-26.

Gilbert LI 2004. Halloween genes encode P450 enzymes that mediate steroid hormone biosynthesis in Drosophila melanogaster. Mol Cell Endocrinol 215: 1-10.

Gutierrez E, Wiggins D, Fielding B, Gould AP 2007. Specialized hepatocyte-like cells regulate Drosophila lipid metabolism. $\mathrm{Na}$ ture 445: 275-280.

Jackson A, Locke M 1989. The formation of plasma membrane reticular systems in the oenocytes of an insect. Tissue Cell 21: 463-473.

Juárez MP 2004. Fatty acyl-CoA elongation in Blattella germanica integumental microsomes. Arch Insect Biochem Physiol 56: 170-178.

Juárez MP, Fernández GC 2007. Cuticular hydrocarbons of triatomines. Comp Biochem Physiol A Mol Integr Physiol 147: 711-730.
Komagata O, Kasai S, Tomita T 2010. Overexpression of cytochrome P450 genes in pyrethroid-resistant Culex quinquefasciatus. Insect Biochem Mol Biol 40: 146-152.

Li X, Schuler MA, Berenbaum MR 2007. Molecular mechanisms of metabolic resistance to synthetic and natural xenobiotics. Annu Rev Entomol 52: 231-253.

Lorenz MW, Anand AN 2004. Changes in the biochemical composition of fat body stores during adult development of female crickets, Gryllus bimaculatus. Arch Insect Biochem Physiol 56: 110-119.

Lycett GJ, McLaughlin LA, Ranson H, Hemingway J, Kafatos FC, Loukeris TG, Paine MJ 2006. Anopheles gambiae P450 reductase is highly expressed in oenocytes and in vivo knockdown increases permethrin susceptibility. Insect Mol Biol 15: 321-327.

Martins GF, Guedes BA, Silva LM, Serrão JE, Fortes-Dias CL, Ramalho-Ortigão JM, Pimenta PF 2011a. Isolation, primary culture and morphological characterization of oenocytes from Aedes aegypti pupae. Tissue Cell 43: 83-90.

Martins GF, Pimenta PF 2008. Structural changes in fat body of $\mathrm{Ae-}$ des aegypti caused by aging and blood feeding. J Med Entomol 45: 1102-1107.

Martins GF, Serrão JE, Ramalho-Ortigão JM, Pimenta PFP 2011 b. Hystochemical and ultrastructural studies of the mosquito Aedes aegypti fat body: effects of aging and diet type. Micro Res Tech, in press.

Müller P, Donnelly MJ, Ranson H 2007. Transcription profiling of a recently colonised pyrethroid resistant Anopheles gambiae strain from Ghana. BMC Genomics 8: 36.

Nene V, Wortman JR, Lawson D, Haas B, Kodira C, Tu ZJ, Loftus B, Xi Z, Megy K, Grabherr M, Ren Q, Zdobnov EM, Lobo NF, Campbell KS, Brown SE, Bonaldo MF, Zhu J, Sinkins SP, Hogenkamp DG, Amedeo P, Arensburger P, Atkinson PW, Bidwell S, Biedler J, Birney E, Bruggner RV, Costas J, Coy MR, Crabtree J, Crawford M, Debruyn B, Decaprio D, Eiglmeier K, Eisenstadt E, El-Dorry H, Gelbart WM, Gomes SL, Hammond M, Hannick LI, Hogan JR, Holmes MH, Jaffe D, Johnston JS, Kennedy RC, Koo H, Kravitz S, Kriventseva EV, Kulp D, Labutti K, Lee E, Li S, Lovin DD, Mao C, Mauceli E, Menck CF, Miller JR, Montgomery P, Mori A, Nascimento AL, Naveira HF, Nusbaum C, O'leary S, Orvis J, Pertea M, Quesneville $\mathrm{H}$, Reidenbach KR, Rogers YH, Roth CW, Schneider JR, Schatz M, Shumway M, Stanke M, Stinson EO, Tubio JM, Vanzee JP, Verjovski-Almeida S, Werner D, White O, Wyder S, Zeng Q, Zhao Q, Zhao Y, Hill CA, Raikhel AS, Soares MB, Knudson DL, Lee NH, Galagan J, Salzberg SL, Paulsen IT, Dimopoulos G, Collins FH, Birren B, Fraser-Liggett CM, Severson DW 2007. Genome sequence of Aedes aegypti, a major arbovirus vector. Science 316: 1718-1723.

Ramalho-Ortigão M, Jochim RC, Anderson JM, Lawyer PG, Pham VM, Kamhawi S, Valenzuela JG 2007. Exploring the midgut transcriptome of Phlebotomus papatasi: comparative analysis of expression profiles of sugar-fed, blood-fed and Leishmaniamajor-infected sandflies. BMC Genomics 8: 300 .

Ramirez JL, Dimopoulos G 2010. The Toll immune signaling pathway control conserved anti-dengue defenses across diverse $A e$. aegypti strains and against multiple dengue virus serotypes. Dev Comp Immunol 34: 625-629.

Romer F, Emmerich H, Nowock J 1974. Biosynthesis of ecdysones in isolated prothoracic glands and oenocytes of Tenebrio molitor in vitro. J Insect Physiol 20: 1975-1987.

Schneider JR, Mori A, Romero-Severson J, Chadee DD, Severson DW 2007. Investigations of dengue-2 susceptibility and body 
size among Aedes aegypti populations. Med Vet Entomol 21: $370-376$

Scott JG, Wen Z 2001. Cytochromes P450 of insects: the tip of the iceberg. Pest Manag Sci 57: 958-967.

Shin SW, Bian G, Raikhel AS 2006. A Toll receptor and a cytokine, Toll5A and Spz1C, are involved in Toll antifungal immune signaling in the mosquito Aedes aegypti. J Biol Chem 281: 39388-39395.

Simpson AE 1997. The cytochrome P450 4 (CYP4) family. Gen Pharmacol 28: 351-359.

Sundseth SS, Nix CE, Waters LC 1990. Isolation of insecticide resistance-related forms of cytochrome P-450 from Drosophila melanogaster. Biochem J 265: 213-217.
Thathy V, Severson DW, Christensen BM 1994. Reinterpretation of the genetics of susceptibility of Aedes aegypti to Plasmodium gallinaceum. J Parasitol 80: 705-712.

Tzou P, De Gregorio E, Lemaitre B 2002. How Drosophila combats microbial infection: a model to study innate immunity and hostpathogen interactions. Curr Opin Microbiol 5: 102-110.

Wicker-Thomas C, Guenachi I, Keita YF 2009. Contribution of oenocytes and pheromones to courtship behavior in Drosophila. BMC Biochem 10: 21.

Wigglesworth VB 1988. The source of lipids and polyphenols for the insect cuticle: the role of fat body, oenocytes and oenocytoids. Tissue Cell 20: 919-932. 